\title{
Loss in Channel Capacity Resulting From Starting Delay in Meteor-Burst Communication
}

\author{
George R. Sugar
}

(March 25, 1960; revised April 25, 1960)

\begin{abstract}
The loss in channel capacity of a meteor-burst communication system is computed as a function of the time required to initiate control of the system. The result is compared with various experimental data and appears to be applicable for signal bursts up to one-half second in duration. It is noted that in the very high frequency range the loss should increase with frequency.
\end{abstract}

\section{Introduction}

It appears that in any practical meteor-burst communication system, there will always be a brief time interval, after the meteor trail is formed, before the channel is used for message transmission. In the case of the familiar controlled two-way systems, this interval consists of one delay associated with the round-trip propagation time, a second associated with the recognition and identification of the distant transmitter, and sometimes a third associated with delays in starting the apparatus itself or in synchronizing one unit with another. In the case of a one-way or broadcast-type system, the propagation delay is not a factor but the other two delays can be significant. Some experimental data on these effects have already been reported by Forsyth et al. [1]. ${ }^{2}$ It is the purpose of this paper to show how the message capacity of a meteor-burst channel is related to a system's initiation time.

\section{Estimation From Detailed Measurements of Burst Statistics}

The loss in channel capacity for a specific system can be estimated directly from measurements of the distribution of signal durations above threshold. The distribution required is not the distribution of meteor-burst lengths in the usual sense but is, rather, the distribution of fading-cycle durations. Each signal excursion above the chosen threshold is assumed to be equivalent to a separate transmission. It is then assumed that signals with durations less than the initiation time are useless for message transmission and that the useful duration of each other signal is reduced by the initiation time. Once the signal duration distribution has been obtained the desired result can be readily computed. As an example, the relation between useful transmission time and system initiation time

1 Contribution from Central Radio Propagation Laboratory, National Bureau of Standards, Boulder, Colo.

2 Figures in brackets indicate the literature references at the end of this paper. obtained from one set of experimental data ${ }^{3}$ is shown in figure 1.

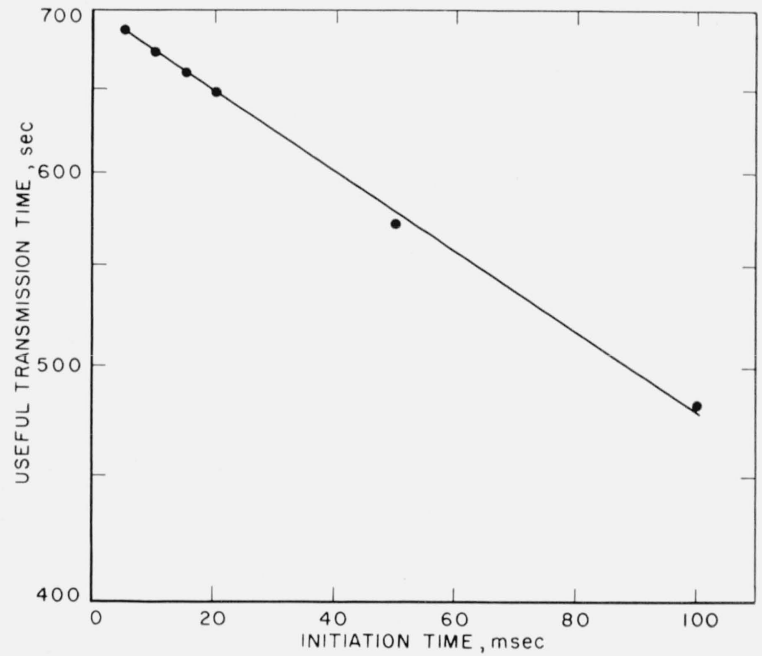

FIgURE 1. Reduction in effective channel capacity as a function of system initiation time.

\section{Estimation From Theory}

An accurate theoretical estimate for the general case appears to be quite complex and will not be attempted at present. Instead only the case for specular underdense trails is considered.

Assume that the signal amplitude for a burst is given by

$$
\begin{array}{ll}
S=0 & \text { for } t<t_{0}, \\
S=S_{0} \exp (-t / \tau) & \text { for } t \geq t_{0},
\end{array}
$$

and that the number $N$ of bursts with peak amplitudes greater than some amplitude $S_{1}$ is given by

$$
N\left(S_{0}>S_{1}\right)=C S_{1}^{-a} .
$$

3 The data used were taken over the transmission path from Long Branch, Ill., to Boulder, Colo., at a frequency near $50 \mathrm{Mc} / \mathrm{s}$. The observing interval 
A burst with duration $t$ at threshold $S_{1}$ then has a peak value $S_{0}=S_{1} \exp (t / \tau)$, and the number of bursts having peak values between $S_{0}$ and $S_{0}+d S_{0}$ is

$$
d N=a C S_{0}^{-a-1} d S_{0} .
$$

The cumulative duration of these bursts is

$$
d T=t d N=\operatorname{taC} S_{0}^{-a-1} d S_{0} .
$$

Upon substituting for $S_{0}$ this becomes

$$
d T=\operatorname{ta} C\left[S_{1} \exp (t / \tau)\right]^{-a-1}\left(S_{1} / \tau\right) \exp (t / \tau) d t .
$$

The total duration $T$ above threshold $S_{1}$ is then

$$
T=\int t d N=C S_{1}^{-a} \int_{0}^{\infty}(a / \tau) t \exp (-a t / \tau) d t,
$$

and

$$
T=(\tau / a) C S_{1}^{-a}
$$

Since this can be written as $T=(\tau / a) N$, the factor $(\tau / a)$ is just the average duration of a burst.

If an initiation time $t_{i}$ is now assumed the useful duration of each burst is decreased and the effective time-above-threshold $T^{\prime}$ becomes

$$
\begin{array}{ll}
T^{\prime}=\int\left(t-t_{i}\right) d N & \text { for } t \geq t_{i} \\
T^{\prime}=0 & \text { for } t<t_{i} .
\end{array}
$$

Upon integration this becomes

or

$$
T^{\prime}=(\tau / a) C S_{1}^{-a} \exp \left(-a t_{i} / \tau\right),
$$

$$
T^{\prime}=T \exp \left(-a t_{i} / \tau\right),
$$

which is the desired result.

It is seen that in a system with an initiation time $t_{i}$ the time available for message transmission is reduced to $\exp \left(-a t_{i} / \tau\right)$ times that available in a system having an initiation time of zero.

\section{Discussion}

We first compare the theoretical relation (5) with two published curves depicting system efficiency in terms of initiation time. Forsyth et al. [1] have published a curve of normalized effective duty cycle versus initiation time, derived partially from operating experience with the JANET system and partly from extrapolation of measurements of signal duration. Their data corresponds nearly exactly (within a few percent) to the relation

$$
T^{\prime}=T \exp \left(-t_{i} / 0.160\right) \text { for } t_{i} \text { in seconds. }
$$

The second curve of interest is that given by Rach [2] for system efficiency versus number of teletype characters omitted (in starting). His curve is based on an experimentally determined distribution of burst durations as applied to a system with a 16.3 msec character length. This curve is nicely described by the relation

$$
T^{\prime}=T \exp \left(-t_{i} / 0.39\right)
$$

A further comparison can be made with data published by Forsyth et al. [ref. 1, fig. 11(c)], giving the distribution of the duration of signal excursions above a fixed threshold. We find that for durations of less than one-half second their curve is of the form $\exp (-t / 0.24)$.

Similar data has been published by Vincent et al. [3] and it is found that for durations of $0.06 \mathrm{sec}$ to 0.6 sec their data are of the form exp $(-t / 0.20)$. (Data for durations less than $0.06 \mathrm{sec}$ are of the form $\exp (-t / 0.04)$. The reason for this discrepancy is not apparent.)

It appears from the above comparisons that for signal durations above threshold of one-half second or less the duration distribution is exponential in form. Therefore, eq (5) is applicable in computing: efficiency as a function of initiation time. Since the theory was based entirely on idealized underdense trails, it is not surprising that the exponential law does not apply for durations longer than one-half second. However, since the experimental data considered the duration of individual excursions above threshold, rather than the total duration of signals from a single trail, it is certain that the fading signals from overdense trails have contributed to the net result. Further study is needed to define the role of the overdense trail in communications applications.

It is interesting to note that the theory predicts a loss in transmission time as the operating frequency increases. Since the conventional form of the time variation of signal amplitude is $S \propto \exp \left(-16 \pi^{2} D t\right.$ $\left.\lambda^{2} \sec ^{2} \phi\right)$, then $\tau \propto \lambda^{2} \sec ^{2} \phi / 16 \pi^{2} D$. Therefore $\tau$ should vary as $\lambda^{2}$. From this it would be expected that the loss would increase rapidly with increase in operating frequency. For example, if we choose $\tau / a=0.2 \mathrm{sec}$ as a representative value at $50 \mathrm{Mc} / \mathrm{s}$ and the initiation time is $40 \mathrm{msec}$, then the channel capacity is reduced to 82 percent of its nominal value. If however the operating frequency were $100 \mathrm{Mc} / \mathrm{s}$, then we would have $\tau / a=50$ milliseconds and the channel capacity would be reduced to 45 percent of its nominal value.

In practice the loss may not be as great as this since, according to Eshleman [4], as the frequency increases the decay constant $\tau$ for underdense trails first decreases and then increases again. However, for a typical communications case, the minimum value appears to occur at frequency of the order of $300 \mathrm{Mc} / \mathrm{s}$ and thus the $\lambda^{2}$ relation should be applicable at frequencies below $100 \mathrm{Mc} / \mathrm{s}$.

\section{References}

[1] P. A. Forsyth, E. L. Vogan, D. R. Hansen, and C. O. Hines, The principles of JANET - a meteor-burst communication system, see figure 16, Proc. IRE 45, 1642 (1957)

[2] R. A. Rach, An investigation of storage capacity required for a meteor-burst communications system, see figure 1, Proc. IRE 45, 1707 (1957).

[3] W. R. Vincent, R. T. Wolfram, B. M. Sifford, W. E. Jaye, and A. M. Peterson, A meteor-burst system for extended range vhf communications, see figure 13, Proc. IRE 45, 1693 (1957).

[4] V. R. Eshleman, Short-wavelength radio reflections from meteoric ionization. Part I: Theory for low-density trails, Scientific Report No. 5 (Radio Propagation Laboratory, Stanford Electronics Laboratories, Stanford Univ., Stanford, Calif., Aug. 30, 1956).

(Paper 64D5-85) 